\title{
Das Selbst entsteht in der Selbsterfahrung
}

\author{
Eckhard Frick
}

Angenommen: 20. Januar 2022 / Online publiziert: 4. Februar 2022

(C) Der/die Autor(en) 2022

Zusammenfassung In diesem Beitrag der Zeitschrift für Psychodrama und Soziometrie setzt sich der Autor mit den Grundlagen der Selbsterfahrung auseinander. „Selbsterfahrung“ ist ein reflexives Nominalkompositum, das aus dem Intensifikator ,selbst-“ und dem nominalisierten Verbum ,erfahren“ zusammengesetzt ist. Das semantische Feld des Kompositums besteht folglich nicht nur in der Reflexivität (Erfahrung der eigenen Existenz), sondern auch in Intensivierung und emphatischer Bekräftigung von Seele und Identität: Das reflexive Kompositum „Selbsterfahrung“ ist die verdichtete Version der reflexiven Proposition „sich erfahren“. Der Prozess sprachlicher Reanalyse, Abstraktion und Verdichtung kann das abstrakte, mit dem bestimmten Artikel als individuelles Konzept gebrauchte Nomen ,Selbst“ entstehen lassen. Dieser sprachliche Prozess vom Handlungsverb zum reflexiven Nominalkompositum und zum abstrakten Nomen drückt aus, was auf der Bühne geschieht: „Role playing is prior to the emergence of the self. Roles do not emerge from the self, but the self may emerge from roles“ (Moreno). Um das Entstehen des Selbst zu verstehen, sollten Psychodramatiker(innen) die Ich-Selbst-Achse im Auge behalten.

Eckhard Frick $(\bowtie)$

Professur für Spiritual Care und psychosomatische Gesundheit, Klinikum rechts der Isar der Technischen Universität München, Kaulbachstr. 22a, 80539 München, Deutschland

E-Mail: eckhard.frick@tum.de 
Schlüsselwörter Selbsterfahrung $\cdot$ Selbst $\cdot$ Ich-Selbst-Achse $\cdot$ Rolle

Individuation · Psychotherapeutische Aus- und Weiterbildung · Psychodrama

\title{
The self emerges in self-experience
}

\begin{abstract}
In this article of the Zeitschrift für Psychodrama und Soziometrie, the author deals with the basics of self-experience. "Selbsterfahrung" (self-experience) is a reflexive nominal compound encompassing as first component the intensifier "selbst-" (X-self) and as second the nominalized verb "erfahren" (experience). Consequently, the compound's semantic domain is not only that of reflexivity (experience of one's own existence) but also of intensification and emphatic assertion of soul and identity: The reflexive compound "Selbsterfahrung" is a condensed version of the reflexive proposition "sich erfahren". The process of linguistic reanalysis, abstraction, and condensation may give rise to the creation of the abstract noun "Selbst" (self), used with the definite article as an individual concept. This linguistic process from verb of action towards reflexive nominal compound and abstract noun expresses what happens on stage: "Role playing is prior to the emergence of the self. Roles do not emerge from the self, but the self may emerge from roles" (Moreno 1946). In order to understand the self's emergence, psychodramatists should focus on the ego-self-axis.
\end{abstract}

Keywords Self-experience $\cdot$ Self $\cdot$ Ego-self-axis $\cdot$ Role $\cdot$ Individuation $\cdot$ Psychotherapy training $\cdot$ Psychodrama

\section{Sprachliche Vorüberlegung: Wie ist der Doppelbegriff „Selbsterfahrung“ gebaut?}

Das Pronomen ,selbst“ kann möglicherweise von *si-liba (,,sein Leib/Leben“: Gebrüder Grimm) abgeleitet werden. Es drückt entweder Identität oder Reflexivität aus:

- Identität: selbst steht in Opposition (Komplementarität) zu ander-: ,J.L. Moreno selbst und kein anderer";

- Reflexivität: selbst steht in Opposition zu einem Personalpronomen: „Sigmund Freud analysiert sich selbst“" vs. ... ihn/sie (Verstärkung des einfachen Reflexivpronomens sich, emphatisch: Intensifikator);

- Hervorgehobene Reflexivität: selbst steht in Opposition zu dem (objektlosen) Nullausdruck ,erfahren“ und betont den Bezug des erfahrenden Subjekts zu sich, im Unterschied zur Erfahrung von Kunst, Musik, Fremdheit usw.

Am reflexiven Gebrauch von selbst wird deutlich, dass dieses Pronomen in schwacher oder starker Betonung verwendet werden kann. Die Hinzufügung von selbst zum Reflexivpronomen sich bringt keine neue Information über den Handelnden, dient aber der Differenzierung gegenüber anderen Akkusativ- oder Dativobjekten eines Verbums und dient der emphatischen Begriffsschöpfung, z. B. „Selbstanalyse“ (Schott 1985) oder „Selbstsupervision“ (Krüger 2017). 
Zur möglichen Betonung des Pronomens „,selbst“ gehört, dass es ebenso wie andere Pronomina (ich, du, man usw.) substantiviert werden kann. Das Selbst steht für die „Menge von Relationen zwischen einem Individuum, das sowohl Objekt als auch Subjekt dieser Relationen ist" (König 2012). Eine weitere sprachliche (emphatische) Möglichkeit stellt die Bildung eines Nominalkompositum (selbst+ nominalisiertes Verb) dar: Das (sich-)Selbst-Erfahren/die Selbsterfahrung. Umgekehrt wird durch Abstraktion aus dem Kompositum das abstrakte Substantiv ,das Selbst“ gewonnen (König 2011).

Das Kompositum „Selbsterfahrung“ lässt sich in die Genitiv-Konstruktion „Erfahrung des Selbst“" umformen, womit gemeint sein kann: (1) das Selbst macht eine Erfahrung (Identität, Genitivus subiectivus); (2) das Selbst wird erfahren (Reflexivität, Genitivus obiectivus).

Gegen die Substantivierung von „selbst“ und anderen Pronomina ist philosophischer Protest angemeldet worden:

Wenn man nun die Wörter „Ich“ und „Selbst“ (groß geschrieben) als Gattungsnamen neu einführt, um den Wesenskern von Menschen zu bezeichnen, führt das zunächst einmal zu sprachlichen Ungereimtheiten. Das Personalpronomen „ich“ kann nun einmal nicht problemlos mit Demonstrativ- und Possessivpronomina sowie mit bestimmten und unbestimmten Artikeln verbunden werden. Dasselbe gilt für die Partikel „,selbst“, „das Ich“, „,ein Ich“, ,,dieses Ich“, ,,mein Ich“, ,das Selbst“, ,ein Selbst“, ,dieses Selbst“, „,mein Selbst“ - das ist zumindest prima facie alles sprachlicher Unsinn! Außerdem führt die Neueinführung von „Ich“ und „Selbst“ dazu, dass man Sätze bilden kann, die nicht nur merkwürdig klingen. (,Ich komme heute Abend zur Party; aber ob mein Ich mitkommt, weiß ich nicht.“ „Natürlich wird die Bundeskanzlerin selbst kommen; aber ihr Selbst lässt sie zu Hause.“) (Beckermann 2021, S. 35).

Diesen philosophischen Bedenken ist der gängige substantivierende Sprachgebrauch (,das Selbst“) entgegenzuhalten, der keineswegs automatisch mit einer Verdinglichung (Reifizierung) psychischer Instanzen oder mit einer substanziellen Verdoppelung einhergehen muss. Es ist aber hilfreich, die zitierte philosophische Kritik an der Substantivierung bei den folgenden Überlegungen im Ohr zu behalten, um vom Selbst nicht als von einer isolierten Substanz zu reden, sondern als von einem relationalen (Beziehungs-)Begriff. Außerdem: Wenn das schwach gebrauchte reflexive selbst ohne Bedeutungsverlust wegfallen kann, also redundant ist, erhebt sich die Frage, ob es zwischen Erfahrung und Selbsterfahrung wirklich eine Differenz gibt, oder ob selbst hier ein Nullausdruck ist, der dem Begriff der Erfahrung äquivalent ist. Wäre dies der Fall, müssten wir hier abbrechen. Weil aber selbst ein breites Spektrum möglicher Betonungen aufweist, auch innerhalb des Doppelbegriffs Selbsterfahrung, setzen wir unsere Überlegungen fort. Wir tun dies im Rahmen der psychodramatischen Anthropologie, die den Menschen in erster Linie als Beziehungswesen, und erst davon abgeleitet als „Individuum“ sieht. Wir können auch sagen: Moreno dreht die Begrifflichkeit um: Erst das soziale Atom (von gr. átomos: „unteilbar“, lat. individuum), dann das Selbst: 
Your self-I don't know how to explain it, but if, God forbid, anything like this ever happens to you, you'll know what I mean. Your self ... is other people, all the people you're tied to, and it's only a thread (Wolfe 2008, S. $547 \mathrm{f}$.).

\section{Selbsterfahrung in der Suche nach professioneller Identität}

Selbsterfahrung als Bestandteil der psychotherapeutischen Identitätssuche geht auf Freuds Forderung einer verpflichtenden Lehranalyse zurück. Freud, der zwar seine „Selbstanalyse“ brieflich mit dem Berliner Hals-Nasen-Ohren-Arzt Wilhelm Fließ diskutierte, jedoch keine „Lehranalyse machte“, schreibt diese Forderung indirekt C.G. Jung zu:

Ich rechne es zu den vielen Verdiensten der Züricher analytischen Schule, daß sie die Bedingung verschärft und in der Forderung niedergelegt hat, es solle sich jeder, der Analysen an anderen ausführen will, vorher selbst einer Analyse bei einem Sachkundigen unterziehen (Freud 1940 [1912], S. 382).

Freud schwankte, welchem Element er Priorität zubilligen sollte:

[...] der Selbstanalyse oder der Lehranalyse. In diesem Schwanken drückt sich die Konfusion zweier Konzepte bei Freud aus: das autodidaktische Konzept der Selbstanalyse und das pädagogische Konzept der analytischen Therapie, das er als „Nacherziehung“ bezeichnet hat (Schott 1985, S. 50).

Nach Laireiter (2015) gilt das Selbsterfahrungsprinzip sowohl in der Verhaltenstherapie als auch in anderen Psychotherapien. Allerdings müsse die Selbsterfahrung im Kontext der übrigen Aus- und Weiterbildung gesehen werden und besser erforscht und evaluiert werden als bisher. Da es keine einheitliche Definition von „Selbsterfahrung“ gebe, schlägt er die folgende Liste vor (Tab. 1).

Laireiter führt eine Reihe von Kontraindikationen und unerwünschten Wirkungen von Lehrtherapien auf, insbesondere übergriffiges oder (sexuell) missbräuchliches Verhalten des Therapeuten oder der Therapeutin. Positiv gewendet, lässt sich sagen, dass die Selbsterfahrung im Rahmen einer Therapie-Ausbildung modellbildend wirkt: Sie bildet - im Guten wie im Schlechten - den Ausgangspunkt für die Entwicklung einer therapeutischen Identität durch möglicherweise lange Berufsjahre.

Tab. 1 Zum Begriff „Selbsterfahrung“ gehörige Phänomene (Laireiter 2015)

Eigen- oder Lehrtherapie der angehenden Psychotherapeut(inn)en
Sensibilisierungstrainings in themenzentrierten Gruppen
Selbstanwendung therapeutischer Methoden in Ausbildungsgruppen oder Ausbildungsseminaren
Feedback und Selbstmodifikation im Rahmen von Ausbildung und Supervision
Selbstreflexion als Komponente der Supervision im Zusammenhang mit der Analyse individueller Antei-
le an der therapeutischen Beziehung
Einübung von therapeutischen Fertigkeiten
Videofeedback und Konfrontation mit sich selbst über dieses Medium


Eine der wenigen empirischen Untersuchungen (Frank et al. 2015) zeigt mit einem mixed-methods-Ansatz, ,dass Selbsterfahrung über alle therapeutischen Orientierungen und Kohorten hinweg nach wie vor als unbedingt notwendiger Ausbildungsbestandteil erlebt wird“, insbesondere, was Persönlichkeitsentfaltung, Kompetenzentwicklung und professionelle Beziehungsgestaltung angeht.

Marks-Tarlow (2010) stützt sich auf die von dem Mathematiker Mandelbrot (1984) begründete rekursive Theorie und interpretiert im Spiel auftauchende SelbstKomplexe als Fraktale. Das Selbst hält er für ein selbst-organisierendes System mit einer fraktalen, sich auf jeweils höheren Ebenen wiederholenden (,rekursiven“) Struktur:

Play helps children create a self as they build an outside world. For the child, this inner and outer construction occurs through the recursive enfolding of feedback gleaned from continual interactions with people and objects. [...] In the typical scenario, a pair of gamers plays several rounds; the success of each player depends upon the choices of the other. During every round, each participant decides anew whether to act cooperatively, which promises big pay-offs but also threatens big losses, or to act selfishly, which offers greater certainties but delivers smaller rewards. The game is recursive, because its feedback-the new information generated by the outcome of each round-gets continually recycled into the system. Through recursion, each round changes the system as a whole while serving as a foundation for the next round (Marks-Tarlow 2010, S. 35).

Die spielerische, insbesondere die psychodramatische Selbsterfahrung greift bereitliegende Selbst-Fraktale auf und gestaltet sie weiter. Selfing (Storch 1999) ist somit kein abgeschlossener Ausbildungs-Baustein, sondern ein dynamischer, rekursiver Prozess.

\section{Rollen- und Selbst-Erfahrung}

Der psychodramatische Rollenbegriff fördert die Dynamik dieses rekursiven Prozesses und schützt vor einem substanzialistischen, identitären Selbstbegriff. Roletaking, Rollentausch und Rollenwechsel, aber auch das Bearbeiten des Rollen-Widerstands im Spielverlauf erfordern Ambiguitätstoleranz, Krappmann (2005, S. 167) zufolge „die für die Identitätsbildung mutmaßlich entscheidendste Variable“:

Ein Individuum, das Ich-Identität behaupten will, muss auch widersprüchliche Rollenbeteiligungen und einander widerstrebende Motivationsstrukturen interpretierend nebeneinander dulden. Die Fähigkeit, dies bei sich und bei anderen, mit denen Interaktionsbeziehungen unterhalten werden, zu ertragen, ist Ambiguitätstoleranz. Sie eröffnet dem Individuum Möglichkeiten zur Interaktion und zur Artikulation einer Ich-Identität in ihr. Aber gleichzeitig ist die Ambiguitätstoleranz auch wieder eine Folge gelungener Behauptung der Ich-Identität, weil sie dem Individuum die Erfahrung vermittelt, auch in sehr widersprüchlichen Situationen die Balance zwischen den verschiedenen Normen und Motiven halten zu können, und dadurch Ängste mindert (Krappmann 2005, S. 155). 
„Rolle“ wird im Alltagssprachgebrauch häufig als etwas sozial Oktroyiertes oder aber als „falsches Selbst“ im Sinne der übernommenen Identität (Marcia 1967) verstanden. J.L. Moreno geht jedoch von der Priorität der Rolle gegenüber der SelbstIdentität aus: Schon als Säuglinge werden wir in ein Handlungs-, Sprach- und SinnSpiel verwickelt, das wir nicht selbst begonnen haben: „Role playing is prior to the emergence of the self. Roles do not emerge from the self, but the self may emerge from roles“ (Moreno 1946, S. 157). Der sprachliche Abstraktionsprozess, innerhalb dessen aus dem reflexiven Nominalkompositum „Selbsterfahrung“ der abstrakte Begriff ,,das Selbst“ entsteht, wird auf der psychodramatischen Bühne inszeniert: Zuerst werden Rollen übernommen, modifiziert, gewechselt und getauscht. Daraus entstehen sekundär Selbstkerne, eine Abfolge von Fraktalen, die dem nie feststellbaren, uns entzogenen Selbst nach und nach ähnlicher werden.

In der Alltagssprache werden ,ich“ und ,selbst“ häufig synonym gebraucht. Differenziert man zwischen beiden, dann wird das Selbst meist als Identitätsbesitz des Sprechers/der Sprecherin aufgefasst. Im Mainstream von Psychoanalyse und Neurobiologie ist das Selbst eine Ich-Repräsentanz, Repräsentation der eigenen Person. Im Gegensatz zur neurowissenschaftlichen Forschung über das Selbst vertreten manche Philosophinnen und Philosophen eine anti-realistische Auffassung. So vertreten Baars (1997) und Metzinger (2009) die konstruktivistische und repräsentationalistische Auffassung, dass unser Gehirn nicht nur die Außenwelt auf einer inneren Bühne auftreten lässt, sondern auch das Selbst, und zwar immer dann, wenn ich den Fokus auf mich als Handelnden, Betroffenen, Erfahrenen usw. legen will. Mit anderen Worten: Wir finden uns in einem permanenten inneren Psychodrama, auf dessen Bühne wir bald die äußere Welt, bald uns selbst, bald andere als Personen erscheinen lassen.

Der Begriff „Person“ (wahrscheinlich vom etruskischen fersu, Äquivalent des griechischen prósōpon) stellt einen wichtigen Aspekt des morenianischen RollenVerständnisses dar: Im antiken Theater ist prósōpon die Maske des Schauspielers, was die Frage aufwirft, wer sich „hinter“ der Maske verbirgt. Auf der virtuellen Bühne ist die Persona ein Avatar oder eine angenommene Identität, die menschliche Akteure nutzen (Metzinger 2018). C.G. Jung verwendet den Persona-Begriff in ähnlicher Bedeutung wie Winnicott (2002 [1960]) das „falsche Selbst“: eine Strategie der sozialen Selbst-Darstellung und Selbst-Erhaltung, die sich den kollektiven Erwartungen anpasst, um das eigene Überleben zu sichern.

Der Gegensatz zwischen philosophischen Anti-Realismen und der positivistischen Selbst-Forschung der Neurowissenschaften machen eine Klärung des Selbst-Begriffs dringend erforderlich. Dan Zahavis (2014) phänomenologische Theorie eines ,experiential self" ist für unseren Kontext deshalb so bedeutsam, weil sie eine Mittelposition zwischen 1. dem Substanzialismus eines unveränderlichen Selbst als neuem Namen für die Seele und 2. der positivistischen Beschreibung von Erfahrungen und Wahrnehmungen bei gleichzeitiger Bestreitung der Existenz einer erfahrenden oder wahrnehmenden Person einnimmt. Zahavis 3. Position setzt beim Verstehen dessen, was das Selbst ist, die Klärung der Erfahrung voraus (und umgekehrt beim Verstehen der Erfahrung die Klärung dessen, was wir unter dem Selbst verstehen): 
Thus, the self currently under consideration - and let us simply call it the experiential self - is not a separately existing entity - it is not something that exists independently of, in separation from, or in opposition to the stream of consciousness - but neither is it simply reducible to a specific experience or (sub)set of experiences; nor is it, for that matter, a mere social construct that evolves through time. Rather, it is taken to be an integral part of our conscious life. More precisely, the claim is that the (minimal or core) self possesses experiential reality and that it can be identified with the ubiquitous first-personal character of the experiential phenomena (Zahavi 2014, Positionen 683-693).

\section{Individuation als Selbst-Erfahrung}

Im Gegensatz zum psychoanalytischen Mainstream sieht C.G. Jung im Selbst nicht eine Ich-Repräsentanz, sondern das Ziel der Individuation, auf das wir zwar lebenslang bezogen sind, das wir jedoch weder messen, identifizieren noch definieren können. Diese Bezogenheit auf das Selbst als unbewussten Archetypus hat Jung zufolge einen ,numinosen“, spirituellen Charakter, jedoch: „Dieses ,Selbst“ steht nie und nimmer an Stelle Gottes, sondern ist vielleicht ein Gefäß für die göttliche Gnade“ (Jung, GW XI, S. 675). C.G. Jungs kongenialer Schüler Erich Neumann hat den lebenslangen Weg der Individuation und Selbst-Werdung entwicklungspsychologisch untersucht und als Ich-Selbst-Achse beschrieben (Frick 2015):

Wenn wir die Persönlichkeit ausschließlich vom Ich her erfassen, können wir sie als eine in einer äußeren Umwelt lebende biopsychische Individualität definieren. Sobald wir aber begriffen haben, dass dieses Ich niemals ohne das ihm zu Grunde liegende Selbst existieren und sich entwickeln kann, kommt es zu der entscheidenden kopernikanischen Wendung in der Tiefenpsychologie, von der aus die menschliche Persönlichkeit und das menschliche Leben nicht mehr vom Ich aus zu verstehen sind, sondern vom Selbst her, um das dieses Ich kreist wie die Erde um die Sonne. Dann aber erkennen wir die Persönlichkeit als eine Wirklichkeit, in welcher die Ich-Selbst-Achse das tragende Phänomen ist. Wir verstehen die Dynamik des menschlichen Lebens als eine Einheit, für die ebenso bewusste wie für das Bewusstsein unbekannte, unbewusste Prozesse und ebenso psychisch ,innere“ wie welthaft ,äußere“ Inhalte einen unauflösbaren Zusammenhang bilden (Neumann 1963, § 575).

Helmut Barz (2003 [1973]), jungianischer Analytiker und Psychodramatiker, betont, dass der Mensch dieses „numinose“, unserem bewussten Zugriff entzogene Selbst ,eben in der ,Selbst"-erfahrung, und das heißt doch: in sich erfährt“ (§ 112). Gegenüber der Abwertung von Selbsterfahrung und Selbstverwirklichung als „EgoTrip“, aber auch gegenüber dem möglichen Missbrauch der Sehnsucht nach Selbsterfahrung betont er:

Ich habe das Streben nach Selbsterkenntnis als eine unausbleibliche Folge des Ich-Bewusstseins aufgefasst und habe dargestellt, dass das Ich im Selbst das 
überindividuelle allgemein Menschliche sucht. Dabei habe ich die Überzeugung vertreten und sie durch einige Beispiele veranschaulicht, dass mit dem Selbst stets auch etwas Numinoses, das heißt eine vom Göttlichen ausgehende Macht erfahren wird. Und ich habe gezeigt, dass dieses numinose Element der Selbsterfahrung in zweierlei Weise lokalisiert werden kann. Entweder wird es als völlig ,extra nos“ (=außerhalb von uns) bestehend angesehen und kommt uns dann nur vom „ganz Anderen“ her entgegen, so dass das Selbst lediglich ein Empfangsorgan für das Göttliche ist, das aber keinerlei direkten Anteil an ihm besitzt; oder dem Selbst wird ein - wenn auch noch so geringer - Eigenanteil am Göttlichen zuerkannt, der dann auch (aber nicht nur) ,intra nos“ (in uns) enthalten ist, wodurch das Selbst zum göttlichen Funken in der menschlichen Seele wird. Dass sich aus diesen beiden entgegengesetzten Auffassungen verschiedene Einstellungen nicht nur zum Göttlichen, sondern auch zum Selbst der Selbsterkenntnis ergeben, liegt auf der Hand (Barz 2004 [1981], § 82).

Die einseitige Betonung des „extra nos“, also der Transzendenz Gottes, kann spirituell zur „Gottesfinsternis“ (Buber 1953), psychisch zur Selbst-Entfremdung führen. Das morenianische, intra nos" heißt: Godplaying, Gott auf die Bühne bringen und so Selbst-Erfahrung zu gestalten - freilich, ohne dadurch die Psychodramatiker(innen) „prophetisch zu überlasten“ (Blattert 2013). Die psychodramatischen Regieprinzipien erlauben es, der Transzendenz des Selbst einen begrenzten Raum auf der Bühne zu geben. Dies ist dann besonders wichtig, wenn das Selbst als göttlich, als numinos aufgeladen erlebt wird. Die Surplus-Reality des Godplaying ermöglicht das Teilen dieser „Immanenz der Transzendenz“ (d.h. Gott auf die Bühne zu bringen) auch dann, wenn die spirituellen Ausrichtungen zwischen Protagonist(in), Leitung und Gruppenmitgliedern differieren.

Wichtige psychodramatische Techniken für das Godplaying sind der leere Stuhl und der Rollentausch mit Gott als Selbst-Erfahrung auf den verschiedenen Intensitäts-Ebenen, die in diesem Beitrag besprochen wurden. Die Ausstrahlung der Technik des leeren Stuhls lässt sich z. B. bei dem Psychoanalytiker Tilmann Moser (2017) beobachten, dessen persönliche Entwicklung von der „Gottesvergiftung zu einem erträglichen Gott“ (Moser 2003) Selbsterfahrung ermöglicht: für ihn selbst und für andere, die sich dem leeren Stuhl annähern.

\section{Fazit in psychodramatischer Perspektive}

Der gängige Selbst-Begriff wird oft individualistisch eingeengt. Demzufolge wird auch "Selbsterfahrung“ isoliert auf das Individuum bezogen, etwa wenn in der psychotherapeutischen Weiter- und Fortbildung „Selbsterfahrung absolviert“ und dementsprechende Punkte und Stunden gesammelt werden, die dem Individuum gutgeschrieben oder auf einem Zeugnis vermerkt werden. Moreno hingegen geht vom sozialen Atom und von der Rolle aus. Folglich ist psychodramatische Selbsterfahrung mehr als (fortbildungs-)punktuelle, individualistische, zertifizierbare Feststellung. Selbst-Erfahrung auf der Bühne ist der Prozess von Begegnung und „Ver- 
anderung“ (Frick 2021): Sie bringt mich dadurch mit mir selbst in Kontakt, dass ich meine isolierte Selbst-Suche loslasse.

Funding Open Access funding enabled and organized by Projekt DEAL.

Open Access Dieser Artikel wird unter der Creative Commons Namensnennung 4.0 International Lizenz veröffentlicht, welche die Nutzung, Vervielfältigung, Bearbeitung, Verbreitung und Wiedergabe in jeglichem Medium und Format erlaubt, sofern Sie den/die ursprünglichen Autor(en) und die Quelle ordnungsgemäß nennen, einen Link zur Creative Commons Lizenz beifügen und angeben, ob Änderungen vorgenommen wurden.

Die in diesem Artikel enthaltenen Bilder und sonstiges Drittmaterial unterliegen ebenfalls der genannten Creative Commons Lizenz, sofern sich aus der Abbildungslegende nichts anderes ergibt. Sofern das betreffende Material nicht unter der genannten Creative Commons Lizenz steht und die betreffende Handlung nicht nach gesetzlichen Vorschriften erlaubt ist, ist für die oben aufgeführten Weiterverwendungen des Materials die Einwilligung des jeweiligen Rechteinhabers einzuholen.

Weitere Details zur Lizenz entnehmen Sie bitte der Lizenzinformation auf http://creativecommons.org/ licenses/by/4.0/deed.de.

\section{Literatur}

Baars, B. J. (1997). In the theatre of consciousness. Global Workspace Theory, a rigorous scientific theory of consciousness. Journal of Consciousness Studies, 4(4), 292-309.

Barz, H. (2003). Selbst-Erfahrung. Tiefenpsychologie und christlicher Glaube. : opus Magnum.

Barz, H. (2004). Stichwort Selbstverwirklichung. Ehrenrettung eines Modewortes. : opus Magnum.

Beckermann, A. (2021). „ich“ und „,elbst“, nicht „Ich“ und „Selbst“. In A. Beckermann (Hrsg.), Aufsätze (Bd. 3, S. 21-42). Bielefeld: Universität Bielefeld.

Blattert, K. (2013). Jakob Levy Moreno - ein Prophet unserer Zeit. Zeitschrift für Psychodrama und Soziometrie, 12(1), 127-181. https://doi.org/10.1007/s11620-013-0209-6.

Buber, M. (1953). Gottesfinsternis. Zürich: Manesse.

Frank, C., Gahleitner, S. B., Gerlich, K., Liegl, G., Hinterwallner, H., Koschier, A., Märtens, M., Schigl, B., Pieh, C., \& Böckle, M. (2015). Selbsterfahrung als Ausbildungsbestandteil der Psychotherapie Qualitätsmerkmal oder Mythos? Zentrale Ergebnisse des Forschungsprojektes „Die Rolle der Selbsterfahrung in der Psychotherapieausbildung“. Resonanzen - E-Journal für biopsychosoziale Dialoge in Psychosomatischer Medizin, Psychotherapie, Supervision und Beratung, 3(2), 114-130.

Freud, S. (1940). Ratschläge für den Arzt bei der psychoanalytischen Behandlung. In A. Freud, E. Bibring, W. Hoffer, E. Kris \& O. Isakower (Hrsg.), Gesammelte Werke (S. 375-387). London: Imago.

Frick, E. (2015). Psychosomatische Anthropologie. Ein Lern- und Arbeitsbuch für Unterricht und Studium (2. Aufl.). Stuttgart: Kohlhammer.

Frick, E. (2021). Veranderte Identität. Zwischen Selbstvergewisserung und Bezogenheit. Stimmen der Zeit, $146(6), 413-423$.

König, E. (2011). Reflexive nominal compounds. Studies in Language. International Journal Sponsored by the Foundation "foundations of Language", 35(1), 112-127.

König, E. (2012). Selbstreflexionen und das Selbst. Sprachliche und konzeptuelle Grundlagen. In G. Gebauer, E. König \& J. Volbers (Hrsg.), Selbst-Reflexionen. Performative Perspektiven (S. 41-58). München: Fink.

Krappmann, L. (2005). Soziologische Dimensionen der Identität. Strukturelle Bedingungen für die Teilnahme an Interaktionsprozessen. Stuttgart: Klett-Cotta.

Krüger, R. T. (2017). Die psychodramatische Selbstsupervision: Wie wir die Idee des spontan-kreativen Menschen in uns selbst verwirklichen können. Zeitschrift für Psychodrama und Soziometrie, 16(2), 273-285. https://doi.org/10.1007/s11620-017-0396-7.

Laireiter, A.R. (2015). Selbsterfahrung. In Verhaltenstherapiemanual (S. 19-23). Berlin Heidelberg: Springer.

Mandelbrot, B. B. (1984). Fractals in physics: squig clusters, diffusions, fractal measures, and the unicity of fractal dimensionality. Journal of Statistical Physics, 34(5), 895-930. 
Marcia, J.E. (1967). Ego identity status: relationship to change in self-esteem. Journal of Personality, 35(1), 118-133.

Marks-Tarlow, T. (2010). The fractal self at play. American Journal of Play, 3(1), 31-62.

Metzinger, T. (2009). Der Ego Tunnel. Eine neue Philosophie des Selbst: Von der Hirnforschung zur Bewusstseinsethik. Berlin: Berlin Verlag.

Metzinger, T. (2018). Why is virtual reality interesting for philosophers? Frontiers in Robotics and AI. https://doi.org/10.3389/frobt.2018.00101.

Moreno, J. L. (1946). Psychosomatic basis and measurement of roles. In J. L. Moreno (Hrsg.), Psychodrama I (S. 157-160). New York: Beacon House.

Moser, T. (2003). Von der Gottesvergiftung zu einem erträglichen Gott. Psychoanalytische Überlegungen zur Religion. Stuttgart: Kreuz.

Moser, T. (2017). Raum für die Neuerfahrung Gottes. Spiritual Care, 6(1), 111-113. https://doi.org/10. 1515/spircare-2016-0146.

Neumann, E. (1963). Das Kind. Struktur und Dynamik der werdenden Persönlichkeit. Zürich: Rascher.

Schott, H. G. (1985). Zauberspiegel der Seele. Sigmund Freud und die Geschichte der Selbstanalyse. Göttingen: Vandenhock \& Ruprecht.

Storch, M. (1999). Identität in der Postmoderne - mögliche Fragen und mögliche Antworten. Allgemeine Heilpädagogik. Eine interdisziplinäre Einführung, 2, 70-84.

Winnicott, D. W. (2002). Ich-Verzerrung in Form des wahren und des falschen Selbst. In D. W. Winnicott (Hrsg.), Reifungsprozesse und fördernde Umwelt (S. 182-199). Gießen: Psychosozial.

Wolfe, T. (2008). The bonfire of the vanities. New York: Picador.

Zahavi, D. (2014). Self and other: exploring subjectivity, empathy, and shame (Kindle-edition). New York: Oxford University Press.

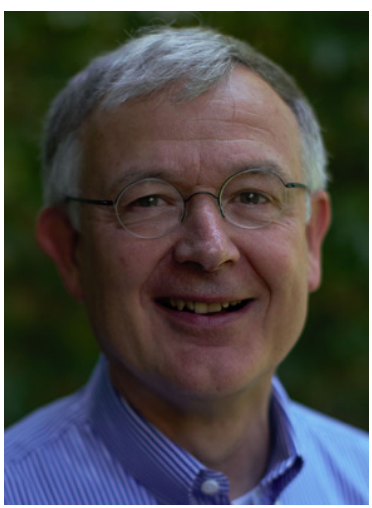

Eckhard Frick Jg. 1955, Prof. Eckhard Frick sj, Professur für Spiritual Care und psychosomatische Gesundheit, Klinik für Psychosomatische Medizin und Psychotherapie, Klinikum rechts der Isar der TU München. Psychodramatiker (Centro Zerka Moreno, Buenos Aires), Lehranalytiker (Münchner Arbeitsgemeinschaft für Psychoanalyse und C.G. Jung Institut München). 